\title{
$\mathbf{J}|\mathbf{A}| \mathbf{C} \mid \mathbf{S}$ \\ $\overline{\text { COMMUNICATIONS }}$
}

Published on Web 08/07/2002

\section{Control of the Anchoring Behavior of Polymer-Dispersed Liquid Crystals: Effect of Branching in the Side Chains of Polyacrylates}

\author{
Jian Zhou, ${ }^{\dagger}$ David M. Collard, ${ }^{\ddagger}$ Jung O. Park, ${ }^{\dagger}$ and Mohan Srinivasarao*,†,‡ \\ School of Textile and Fiber Engineering and School of Chemistry and Biochemistry, \\ Georgia Institute of Technology, Atlanta, Georgia 30332
}

Received June 4, 2002

Nematic liquid crystal (LC) fluids in contact with solid substrates often adopt a preferred direction of alignment, referred to as anchoring. Transitions from one alignment to another, for example from normal alignment (homeotropic) to parallel alignment (planar), are referred to as anchoring transitions. Temperature-driven anchoring transitions usually occur at temperatures $\left(T_{\mathrm{t}}\right)$ close to those of bulk phase transitions ${ }^{1}$ (e.g., the nematic-isotropic transition temperature, $T_{\mathrm{NI}}$ ). Wide separation of the anchoring and bulk phase transition temperatures is rare. Amundson and Srinivasarao reported a set of temperature-driven anchoring transitions of nematic LC at polymer interfaces created by photopolymerization-induced phase separation. ${ }^{2} T_{\mathrm{t}}$ was tuned, independent of the bulk phase transitions, by varying the length of the linear side chain of the polyacrylate matrix. It was postulated that microscopic interactions between the side chain of the polyacrylate and LC molecules dominate the anchoring behavior. A thermodynamic explanation was proposed. ${ }^{2}$ Here we report the effect of incorporating a methyl branch into the side chain of polyacrylates on the anchoring behavior of polymer-dispersed liquid crystal (PDLC) films. A series of poly(methylheptyl acrylates) were used as polymer matrixes (Table 1). Nematic anchoring at the interface of poly(1-methylheptyl acrylate) is dramatically different from that of the other polyacrylates with the methyl branch farther removed from the polymer backbone. These results suggest that the different conformation of the side chains of the polyacrylates is an important factor in determining the mode of anchoring.

Methylheptyl acrylate (MHA) monomers were synthesized from the corresponding alcohols and acryloyl chloride. ${ }^{3}$ The homopolymer of each monomer was prepared by radical polymerization in solution. ${ }^{4}$ The glass transition temperatures $\left(T_{\mathrm{g}}\right)$ of these amorphous homopolymers $\left(M_{\mathrm{w}}=75000-90000 \mathrm{~g} \mathrm{~mol}^{-1}\right.$ and $M_{\mathrm{w}} / M_{\mathrm{n}}=3.0-$ 4.0 , determined by gel permeation chromatography) were measured using differential scanning calorimetry (Seiko DSC 220) at a heating rate of $10^{\circ} \mathrm{C} / \mathrm{min}$. PDLC films were prepared using nematic fluid TL205 (EM Industries), a mixture of halogenated bi- and terphenyls with aliphatic tails of 2-5 carbons in the 4-position of the phenyl group. This fluid displays a nematic phase over a wide temperature range $\left(-20\right.$ to $87^{\circ} \mathrm{C}$ ), thereby allowing the exploration of the anchoring behavior over a temperature range greater than that possible for single-component nematics. ${ }^{2}$ A mixture of TL205 (80.0 wt $\%)$, alkyl acrylates ( $17.8 \mathrm{wt} \%)$, 1,1,1-trimethylyol propane triacrylate $(2 \mathrm{wt} \%)$, and photoinitiator $(0.1-0.2 \mathrm{wt} \%$, Darocur 1173 , Ciba) was placed in a glass cell with a gap of 5 or $15 \mu \mathrm{m}$. The triacrylate is used as a cross-linking agent that provides rigidity to the PDLC structure. The mixture was irradiated by UV light

\footnotetext{
* Address correspondence to this author. E-mail: mohan.srinivasarao@ textiles.gatech.edu.

$\dagger$ textiles.gatech.edu.
$\dagger$ School of Textile and Fiber Engineering.

$¥$ School of Chemistry and Biochemistry.
}

Table 1. Anchoring Transition Temperatures $\left(T_{t}\right)$ of TL205 in PDLC ${ }^{a}$ and Glass Transition Temperatures $\left(T_{g}\right)$ of Homopolymers

\begin{tabular}{|c|c|c|c|}
\hline Acrylates & Monomer structure & $\begin{array}{c}T_{t} \\
\left({ }^{\circ} \mathrm{C}\right)\end{array}$ & $\begin{array}{c}\text { Homopolymer } \\
T_{g}\left({ }^{\circ} \mathrm{C}\right)\end{array}$ \\
\hline $\begin{array}{l}\text { n-heptyl } \\
\text { acrylate }\end{array}$ & & 78 & $-60^{b}$ \\
\hline 1-MHA ${ }^{c}$ & & $\smile^{d}$ & $-46^{b}$ \\
\hline 2-MHA & & 78 & -60 \\
\hline 3-MHA & & 71 & -65 \\
\hline 4-MHA & & 73 & -62 \\
\hline 5-MHA & & 71 & -58 \\
\hline
\end{tabular}

${ }^{a} T_{\mathrm{NI}}$ of all of the PDLC films here is $84^{\circ} \mathrm{C}$. Based on the phase diagram, this corresponds to $\sim 1 \%$ residual monomer in the LC phase at the end of photopolymerization. ${ }^{b}$ Compare to ref $15 .{ }^{c}$ MHA represents methylheptyl acrylate. ${ }^{d}$ Only planar anchoring was observed down to $-14{ }^{\circ} \mathrm{C}$; see text.

(365 nm) using a mercury lamp and a dichroic filter. This mixture is initially a homogeneous and isotropic phase, but phase separation occurs during photopolymerization to form LC-rich and polymerrich phases. Films with cellular morphology were made by a twostep photopolymerization: a slow polymerization process induced by a UV irradiation with low intensity $\left(40 \sim 100 \mu \mathrm{W} / \mathrm{cm}^{2}\right)$ for several hours, followed by curing at higher intensity $\left(0.5 \mathrm{~mW} / \mathrm{cm}^{2}\right)$ for 20 min. The size of the LC cells can be varied from 5 to $70 \mu \mathrm{m}$ by changing polymerization conditions. The morphology of the PDLC films was visualized in three dimensions using a Leica TCS-SP confocal microscope. ${ }^{5,6}$ Pyrromethene 546 (Exciton, Inc., $1 \times 10^{-4}$ mole fraction) was added to the polymerization mixture to provide the imaging contrast between the two phases. The dye was excited at $488 \mathrm{~nm}$ by an $\mathrm{Ar}^{+}$laser, and the fluorescence emission was collected from 515 to $580 \mathrm{~nm}$. The long axis of the dye is expected to align parallel to the director of nematic LC. ${ }^{7}$ The slow polymerization process leads to a cellular morphology, which contains only one layer of close-packed polygonal LC cells between the two glass substrates, as shown in Figure 1. Each LC cell is completely enclosed by a polymeric matrix that extends through the film, with thin vertical polymer walls from the top substrate to the bottom. Such cellular morphology facilitates identifying the anchoring present in the PDLC films.

Anchoring behavior of the PDLC films was evaluated using a polarized light microscope equipped with a hot stage. An example of a homeotropic-to-planar transition (H-to-P) is shown in Figure 2. For a film made from 2-MHA/TL205, a uniform homeotropic 

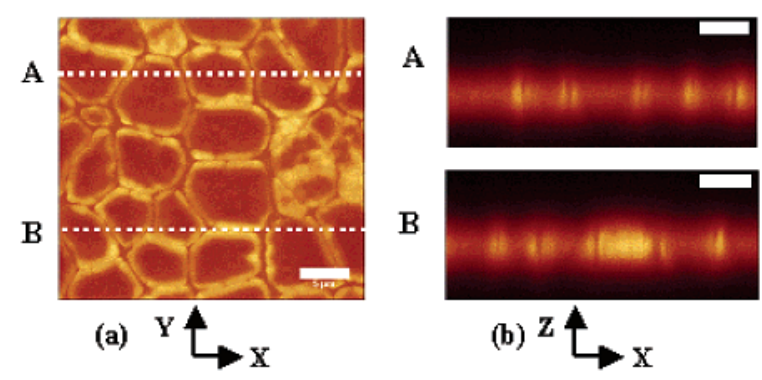

Figure 1. Confocal microscopic sections of a cellular PDLC film with homeotropic anchoring: (a) $X Y$ image and (b) $X Z$ images taken along the dotted lines in (a). The polarization of excitation is along the $Y$ direction. The scale bar is $5 \mu \mathrm{m}$.

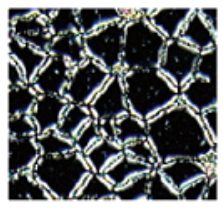

(a) $70^{\circ} \mathrm{C}$

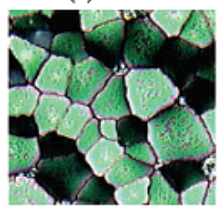

(c) $78^{\circ} \mathrm{C}$

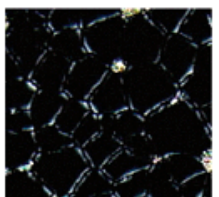

(b) $76^{\circ} \mathrm{C}$

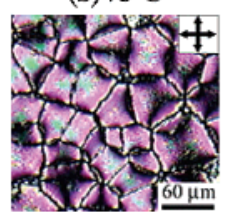

(d) $81^{\circ} \mathrm{C}$

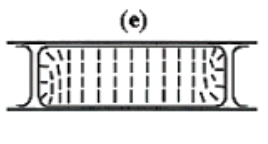

(f)

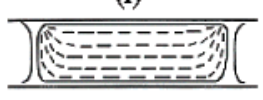

Figure 2. Polarized light micrographs of the homeotropic-to-planar anchoring transition of a PDLC film made from 2-MHA/TL205. (a) and (b) are for homeotropic anchoring and (c) and (d) are for planar anchoring. The schematics, (e) and (f), represent possible director fields within a LC cell with homeotropic and planar anchoring, respectively.

anchoring is observed at temperatures below $T_{\mathrm{t}}$. The centers of the LC cells are dark under crossed-polarizers, indicating that the optic axis of the LC is along the propagation direction of the light. The edges of the polymer walls appear the brightest if the projection of the wall is aligned at about $\pm 45^{\circ}$ to the polarizer or analyzer, consistent with homeotropic anchoring at the polymer walls. One of the possible director configurations in a LC cell with homeotropic anchoring is shown in Figure 2e. When the temperature is increased to $T_{\mathrm{t}}$, the appearance of the film changes dramatically such that in-plane birefringence dominates the texture (Figure 2c). When the temperature is $3{ }^{\circ} \mathrm{C}$ above $T_{\mathrm{t}}$ (Figure $2 \mathrm{~d}$ ), anchoring with a tilt angle less than $20^{\circ}$ (close to planar anchoring) is estimated from the orientation of the dark brushes of the nematic texture near the polymer walls. A weak anchoring condition is observed when the temperature is close to $T_{\mathrm{t}}$, as shown by very uniform alignment of the nematic molecules within each LC cell in both Figure $2 b$ and $2 c{ }^{8}$

The values of $T_{\mathrm{t}}$ for various acrylates are shown in Table 1 . The error in measuring $T_{\mathrm{t}}$ is ca. $\pm 0.5^{\circ} \mathrm{C}$. The most striking observation is that poly(1-MHA) induces only planar anchoring, even if the temperature is as low as $-14{ }^{\circ} \mathrm{C}$ (i.e., no anchoring transition was observed), while all other poly(MHAs) show H-to-P transitions at $71-78{ }^{\circ} \mathrm{C}$. Since $T_{\mathrm{t}}$ is far above $T_{\mathrm{g}}$ of the polyacrylates (Table 1 ), and insensitive to the amount of the cross-linking agent (and thereby to the average length of the polymer chains between the crosslinking points) within the range studied, mobility of the polymer backbone is ruled out as the origin of the anchoring transitions. $T_{\mathrm{t}}$ is also insensitive to the size of the LC domains within the range of $5-70 \mu \mathrm{m}$. In contrast, $T_{\mathrm{t}}$ is very sensitive to the variation of the structure of the side chain. PDLC films were also made from a single-component LC, 4- $n$-pentyl-4'-cynobiphenyl (5CB), and the acrylates in Table 1. Anchoring of $5 \mathrm{CB}$ is similar to that of TL205: the film prepared with 5CB and 1-MHA shows only planar anchoring, while the films prepared with the other acrylates show H-to-P transitions. ${ }^{9}$

To explain these observations, we consider an interfacial model that accounts for enthalpic and entropic contributions to the anchoring transition. Van der Waals interaction between the side chains of polyacrylates and the alkyl tails of LC molecules provides the enthalpic drive for homeotropic anchoring. ${ }^{\mathrm{a}, 2,10} \mathrm{NMR}$ investigation ${ }^{11}$ has shown that the alkyl side chains of polyacrylate contacting the nematic molecules at the interface are partially ordered under homeotropic anchoring conditions. On the other hand, planar anchoring is driven by the entropy of the interfacial free energy, which is contributed by both the entropy-favored planar packing of the rodlike molecules on the surface ${ }^{12}$ and the order-disorder transition of the side chains. ${ }^{13}$ The two driving forces are competitive and reach a balance at $T_{\mathrm{t}}$. We suggest that the different behavior of the 1-methylheptyl side chain in anchoring is related to the dramatic difference in the side chain conformation. For poly(1MHA), where the methyl substituent is closest to the polymer backbone, the side chain is likely to adopt a "tilted" conformation with respect to the interface normal, such that an ordered packing of the side chains at the interface is impossible. Planar anchoring is thus preferred even at temperatures as low as $-14{ }^{\circ} \mathrm{C}$ for TL205. This suggestion is consistent with the observation that the $T_{\mathrm{g}}$ of poly(1-MHA) is $\sim 15^{\circ} \mathrm{C}$ higher than that of the other polyacrylates (though $T_{\mathrm{g}}$ is not directly related to the anchoring transition discussed here). It is also interesting to note that 1-methylheptyl as a substituent in LC molecules usually has a strong effect on the packing of the molecules in the mesophase. ${ }^{14}$

In summary, we have demonstrated that the position of the methyl substituent along the alkyl side chain of polyacrylates affords control of the anchoring of nematic fluids in PDLC films. In particular, when the methyl substituent is closest to the polymer backbone, it dramatically changes the anchoring of the nematic phase confined in the polymer matrix. We propose that the conformation of the side chain is responsible for the observed anchoring behavior.

Acknowledgment. This work was supported by the National Science Foundation (CAREER) under Grant No. 0096240. We thank M. Vargas and F. Salhi for help in the synthesis of monomers.

\section{References}

(1) (a) Jerome, B. Rep. Prog. Phys. 1991, 54, 391-451. (b) Yokoyama, H. In Handbook of Liquid Crystal Research; Collings, P. J., Patel, J. S., Eds. Oxford University Press: New York, 1997; Chapter 6. (c) Patel, J. S.; Yokoyama, H. Nature 1993, 362, 525-527. (d) Founier, J. B.; Galatola P. Phys. Rev. Lett. 1999, 82, 4859-4862.

(2) Amundson, K. R.; Srinivasarao, M. Phys. Rev. E 1998, 58, R1211-R1214.

(3) Shaughnessy, K. H.; Kim, P.; Hartwig, J. F. J. Am. Chem. Soc. 1999, $121,2123-2132$.

(4) Guo, X. A.; Hunter, A. D.; Chen, J. J. Polym. Sci., Part A: Polym. Chem. 1994, 32, 47-56.

(5) Amundson, K. R.; Blaaderen, A. V.; Wiltzius, P. Phys. Rev. E. 1997, 55 $1646-1654$.

(6) Song, Y.; Srinivasarao, M.; Tonelli, A.; Balik, C. M.; McGregor, R. Macromolecules 2000, 33, 4478-4485.

(7) Karolin, J.; Johansson, L. B.-A.; Strandberg, L.; Ny, T. J. Am. Chem. Soc. 1994, 116, 7801-7806.

(8) Drzaic, P. S. Liquid Crystal Dispersions; World Scientific: Singapore, $1995 ;$ p 150.

(9) However, for the $5 \mathrm{CB}$ films, $T_{\mathrm{t}}$ is close to $\left(1-2{ }^{\circ} \mathrm{C}\right.$ below) $T_{\mathrm{NI}}$ of the films, and the H-to-P transition is likely due to prewetting (refs $1 \mathrm{a}, \mathrm{b}$ ).

(10) Porte, G. J. Phys. (France) 1976, 37, 1245-1252.

(11) Roussel, F.; Canlet, C.; Fung, B. M. Phys. Rev. E 2002, 65, 021701.

(12) Sharlow, M. F.; Gelbart, W. M. Liq. Cryst. 1992, 11, 25-30.

(13) Aliev, F. M.; Zgonnik, V. N. Eur. Polym. J. 1991, 27, 969-973.

(14) (a) Walba, D. M.; Korblova, E.; Shao, R.; Maclennan, J. E.; Link, D. R.; Glaser, M. A.; Clark, N. A. Science 2000, 288, 2181-2184. (b) Collard, D. M.; Lillya, C. P. J. Am. Chem. Soc. 1991, 113, 8577-8583.

(15) Andrews, R. J.; Grulke, E. A. In Polymer Handbook; Brandrup, J., Immergut, E. H., Grulke, E. A., Eds.; John Wiley and Sons: New York, 1999; VI-200.

JA027162A 